\title{
Detection of Slippery Terrain with a Heterogeneous Team of Legged Robots
}

\author{
Duncan W. Haldane*, Péter Fankhauser*, Roland Siegwart, and Ronald S. Fearing
}

\begin{abstract}
Legged robots come in a range of sizes and capabilities. By combining these robots into heterogeneous teams, joint locomotion and perception tasks can be achieved by utilizing the diversified features of each robot. In this work we present a framework for using a heterogeneous team of legged robots to detect slippery terrain. StarlETH, a large and highly capable quadruped uses the VelociRoACH as a novel remote probe to detect regions of slippery terrain. StarlETH localizes the team using internal state estimation. To classify slippage of the VelociRoACH, we develop several Support Vector Machines (SVM) based on data from both StarlETH and VelociRoACH. By combining the team's information about the motion of VelociRoACH, a classifier was built which could detect slippery spots with $92 \%(125 / 135)$ accuracy using only four features.
\end{abstract}

\section{INTRODUCTION}

Versatile locomotion over all types of terrain is one of the goals of legged robotics. While a great amount of work has been presented for legged locomotion on solid grounds, safe and fast handling of slippery terrain is still an open research problem. The biggest challenge of slippery terrain presents its inability to be detected without physical contact. Estimating the slipperiness through contact on a step-by-step basis is an extremely slow process. For these reasons, we have chosen the alternative approach of deploying a group of robots. A robot team is more capable of successfully fulfilling a task than a single robot in many aspects. For example, a task can be distributed amongst the team members which lowers the constructional and control complexity for the individual robots. Furthermore, with parallelization, the problem be solved faster, and a redundancy in the team allows the task to be executed more robustly.

With these advantages in mind, we present a framework for a small heterogeneous team of legged robots. Our goal is to navigate a relatively large and more capable robot, the main robot, through an area with slippery regions. These slippery patches are potentially hazardous and the main robot needs to avoid them in order to protect the sensitive

This material is based upon work supported by the National Science Foundation under IGERT Grant No. DGE-0903711, and Grant No. CNS0931463, and the United States Army Research Laboratory under the Micro Autonomous Science and Technology Collaborative Technology Alliance.

This work was supported in part by the Swiss National Science Foundation (SNF) through project 200021_149427 / 1 and the National Centre of Competence in Research Robotics.

D.W. Haldane is with the Department of Mechanical Engineering, University of California, Berkeley, CA 94720 USA, dhaldane@berkeley.edu

P. Fankhauser and R. Siegwart are with the Autonomous Systems Lab (ASL), ETH Zurich, Switzerland,pfankhauser@ethz.ch,rsiegwart@ethz.ch

R.S. Fearing is with the Department of Electrical Engineering and Computer Sciences, University of California, Berkeley, CA 94720 USA, ronf@eecs.berkeley.edu

* These authors contributed equally to this work.

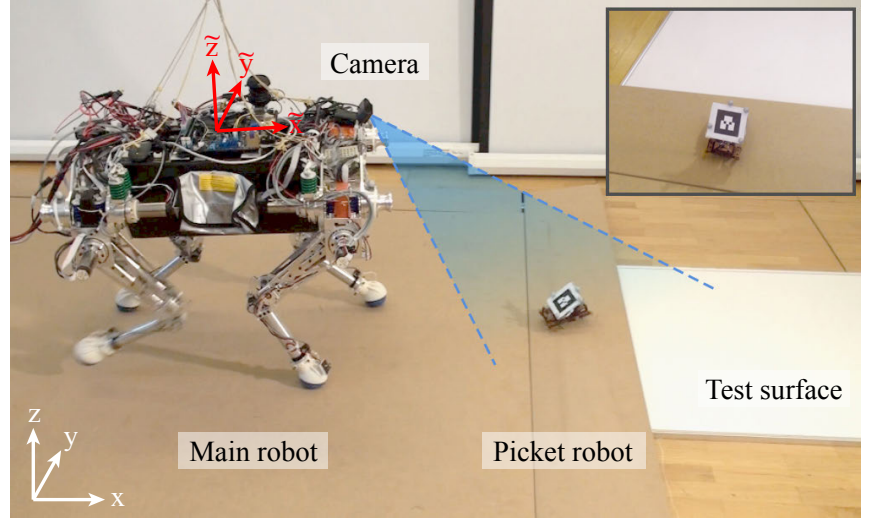

Fig. 1: Our proof of principle setup consists of the main robot StarlETH and one picket robot VelociRoACH. A camera on the main robot tracks the picket robot and allows to guide it at a constant distance of $0.5 \mathrm{~m}$ ahead. The test surface is a whiteboard which is either left dry or made slippery with lubricant.

and expensive onboard equipment. We can achieve this by sending multiple smaller robots, the picket robots, ahead of the main robot. The picket robots assess of the area in front of the main robot so that a safe path can be chosen. These smaller robots are simpler in construction and cheaper; therefore, a loss of a picket robot is tolerable. Due to the limited capabilities of the picket robots, they depend on localization and guidance assistance from the main robot. This collaborative work of the heterogeneous team of legged robots enables to safely navigate the main robot through a field with hazardous slippery regions without putting restrictions on its locomotion speed.

To apply this approach, many topics need to be addressed. Each robot needs to be able to traverse the terrain autonomously, the robots need to communicate, localize themselves as a group and relatively to the environment, and plan and execute a route while probing, mapping and avoiding dangerous regions. In this work, we focus on tackling the task of detecting slippery areas and localizing the probing robot from the main robots perspective. We save the diverse tasks of coverage planning, mapping and navigation for future work.

\section{A. Prior Work}

There have been many approaches to terrain classification using techniques which can be divided into two categories: Remote sensing using cameras or radar, and vibration based classification. For planning purposes, it is desirable to have information about terrain before the robot encounters it. To this end, terrain classification techniques using 3D-point 
clouds [27] or visual data [5] have been developed. These methods require complex sensing apparatus, such as cameras or laser range finders, and are largely dependent on the presence of texture (visual or physical) in the dataset. An alternative, vibration-based terrain classification, uses simple sensors such as accelerometers or gyroscopes to detect terrain using characteristic vibratory signatures ([29], [28], [26], [10], [12]). The disadvantage of the vibration-based approach is that the robot must be physically present on the terrain, which might be hazardous. To avoid stepping on the terrain, several classification methods use an appendage to identify properties of directly adjacent terrain([15], [25], [13]), which limits the planning horizon for navigation. The goal of the present work is to be able to remotely classify terrain which may be devoid of texture, without risking a valuable robot. An example of such terrain is a smooth surface which has lubricated regions. These spots look visually identical, and have no physical texture which could discriminate them. More examples include hidden holes and troughs, and hazards obscured by leaf litter.

Different forms of heterogeneous mobile robot teams have been introduced in the last years. They are varied in aspects such as team architecture, task assignment, communication, and localization (see [23] for an overview). Our approach is similar to work in [20] where a bigger and more intelligent main robot assists smaller and less capable robots (picket robots) for navigation. In return, the small sensor robots can deliver information from areas that are unaccessible or dangerous for the bigger robot. Similarly, in [8] a big wheeled vehicle was supporting smaller quadruped robots in a search and rescue scenario. For a successful collaborative navigation a precise localization strategy is required. The work of [21] has demonstrated assistive navigation with vision based marker detection and pose estimation. However, the chosen fiducial markers restrict to planar pose estimation. A marker-free, model-based tracking algorithm for cooperative robots was presented in [19], which requires a stereo camera setup. So far, little attention has been given to heterogeneous teams involving legged robots. Besides the work of [8], an exception to this is the research in [22] where the six-legged robot Genghis-II was used collaboratively to push boxes with a wheeled vehicle.

\section{B. Approach}

As a proof of concept, we restrict our robot team to one main robot and one picket robot; many other team configurations are possible. For navigation, we use the inverse of the "Follow the leader" approach, wherein the main robot drives the picket robot using constant position feedback. The picket robot assesses the terrain in front of the main robot by using a vibration-based terrain classifier. In Section IIA, we describe our experimental setup. The main robot, and its sensing capabilities are described in Sections II-B and II-D. The picket robot is described in Section II-C, and the classification approach is described in Section IIE. The efficacy of the main robot's localization approach is evaluated in Section III-A, and the accuracy of our classifiers

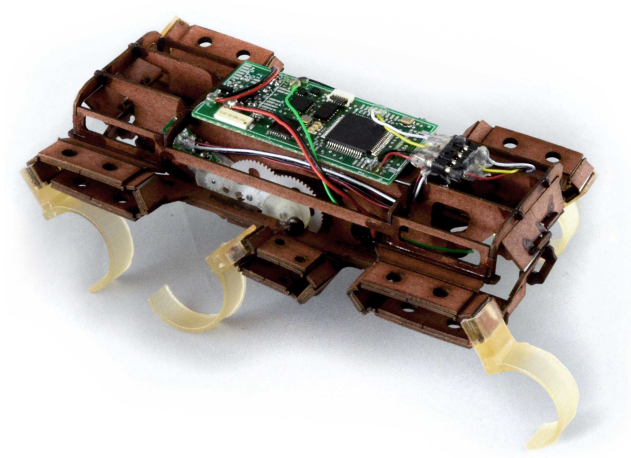

Fig. 2: The picket robot, VelociRoACH. This hexapedal robot is $10 \mathrm{~cm}$ long, weighs $35 \mathrm{~g}$ and is powered by two DC brushed motors [14].

is given in Section III-B. The results from this work are summarized in the accompanying video ${ }^{1}$.

\section{Methods}

\section{A. Overview of the Setup}

We evaluated our methods in a laboratory environment as shown in Fig. 1. The setup consists of the main robot and one picket robot characterizing the ground slipperiness. The test surface is a whiteboard $(1.2 \times 0.75 \mathrm{~m})$ which is either left dry (coefficient of friction $\mu=0.39$ ) or is sprayed uniformly with a silicone-oil-based release agent ${ }^{2}$, making the surface slippery $(\mu=0.14)$. The main robot runs an on-board state estimation and carries a downward-facing camera to track the smaller robot in front of it. The combination of on-board state estimation and visual tracking allows the main robot to steer and to localize the picket robot. The estimated pose and the desired position of the picket robot is shared between the robots via $\operatorname{ROS}^{3}$ messages over 802.15.4 radio. We quantify the performance of the pose estimation system by comparing the data with the ground truth provided by an external optical tracking system.

\section{B. The Main Robot}

The quadruped StarlETH [17] is used as the main robot, which has the shape and weight of a medium-sized dog. In addition to its onboard electronics and power supply, StarlETH is able to carry a payload of $\sim 15 \mathrm{~kg}$, which is sufficient for highly accurate perception sensors. All legs of the system are fully torque controllable and allow the robot move in a variety of different gaits. In our experiments, StarlETH uses a static walking gait [11], which is robust against (unperceived) terrain variations and external disturbances. The desired global travel direction (speed and heading) is controlled manually with a joystick. For state estimation, StarlETH fuses kinematic data from the legs with on-board Inertial Measurement Unit (IMU) measurements [3]. The algorithm is able to estimate the position of all footholds and the 6 DoF pose of the main body without prior knowledge on the geometrical structure of the terrain.

\footnotetext{
${ }^{1}$ Also available at http://youtu.be/3LDXy5RVAbU

${ }^{2}$ Pol-Ease 2300 from Polytek

${ }^{3}$ Robot Operating System
} 


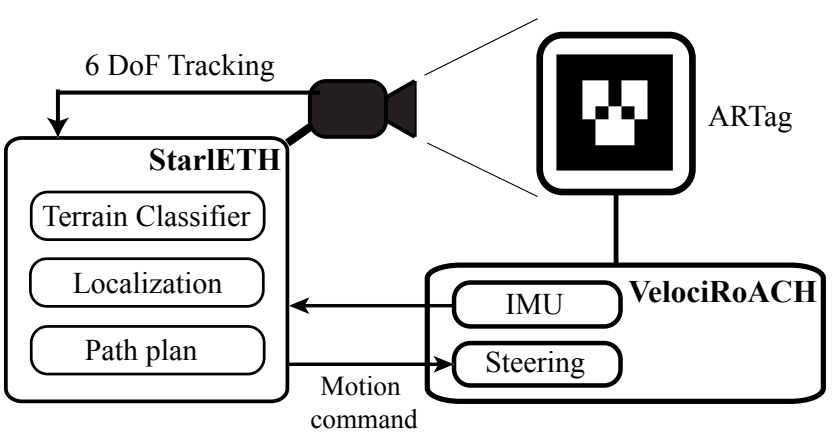

Fig. 3: A block diagram showing the localization and terrain classification information flows between the members of the robot team.

\section{The Picket Robot}

Our joint terrain detection framework makes most sense if the picket robot is a cheap and robust robot that is capable of traversing terrain at least at the speed of the main robot. Smaller robots can be more robust as an effect of size [18], and can be cheaper than larger robots by several orders of magnitude. We chose the VelociRoACH as our picket robot because it fulfills these criteria. The VelociRoACH [14] is a hexapedal millirobot (shown in Fig. 2) built with cardboard Smart Composite Microstructures [16], making it cost efficient to produce. It is $10 \mathrm{~cm}$ long, capable of traversing rough terrain, and has a top speed of $2.7 \mathrm{~m} / \mathrm{s}$.

The VelociRoACH is driven by the imageproc ${ }^{4}$ [2] robot control board. The imageproc also collects telemetry data at $1000 \mathrm{~Hz}$, and uses a 802.15 .4 radio interface for communication and external control ${ }^{5}$.

The main robot drives the picket robot in front of it at a distance of $0.5 \mathrm{~m}$ to detect slippery patches of terrain. We used the following control law to prescribe the desired motion of the VelociRoACH:

$$
\begin{aligned}
& \dot{\tilde{x}}_{\mathrm{des}}=K_{p, x}\left(\tilde{x}_{\mathrm{des}}-\tilde{x}\right), \\
& \dot{\tilde{\psi}}_{\mathrm{des}}=K_{p, y}\left(\tilde{y}_{\mathrm{des}}-\tilde{y}\right)+K_{p, \psi}\left(\tilde{\psi}_{\mathrm{des}}-\tilde{\psi}\right),
\end{aligned}
$$

where $\dot{\tilde{x}}_{\mathrm{des}}$ is the desired forward velocity, $\tilde{x}_{\mathrm{des}}$ and $\tilde{x}$ are the target and actual distances to StarlETH, respectively. Similarly, $\dot{\tilde{\psi}}_{\text {des }}$ is the desired yaw rate, $\tilde{y}_{\text {des }}$ and $\tilde{y}$ are the target and actual distances from the midline of the StarlETH, respectively, and $\tilde{\psi}_{\text {des }}$ and $\tilde{\psi}$ are the target and actual yaw angles, respectively.

To steer the VelociRoACH, we assume that differential steering dynamics apply and drive the two sides of the VelociRoACH at different speed to achieve turning (as was done in Buchan and Haldane et. al. [4]). The desired leg speeds for the left and right side, $\dot{\alpha}_{l, \mathrm{des}}, \dot{\alpha}_{r \text {,des }}$ are given by

$$
\left[\begin{array}{c}
\dot{\alpha}_{l, \mathrm{des}} \\
\dot{\alpha}_{r, \mathrm{des}}
\end{array}\right]=\frac{1}{r}\left[\begin{array}{cc}
1 & d / 2 \\
1 & -d / 2
\end{array}\right]\left[\begin{array}{c}
\dot{x}_{\mathrm{des}} \\
\dot{\psi}_{\mathrm{des}}
\end{array}\right],
$$

where $r$ is the effective leg radius, and $d$ is the width of the robot.

\footnotetext{
${ }^{4}$ Embedded board: https://github.com/biomimetics/imageproc_pcb

${ }^{5}$ Embedded code: https://github.com/dhaldane/roach
}

The control loop on position and orientation (from StarlETH's camera to VelociRoACH) is closed at $30 \mathrm{~Hz}$. Internally, the VelociRoACH uses PID feedback control at $1000 \mathrm{~Hz}$ to regulate the speed of its legs.

\section{Visual Tracking}

The localization of the picket robot is performed by visually tracking a fiducial marker attached to the robot. The camera is mounted at a fixed position on the front of the main robot at a height of $0.5 \mathrm{~m}$ and the viewpoint is pointed in the direction of travel and downwards at an angle of $30^{\circ}$ (see Fig. 1). This created a distance of $\sim 1 \mathrm{~m}$ from camera to marker, depending on the relative position of the main and picket robot. The camera is a commercial webcam ${ }^{6}$ used at a resolution of $640 \times 480 \mathrm{px}$. The marker is an ARTag [9] (side length $6 \mathrm{~cm}$ ) and we use the ALVAR software library [24] to track the pose of the marker relative to the camera (and hereby to the main robot). This setup allows for realtime tracking of the picket robot's full 6 DoF relative to the camera. Together with the state estimation of our main robot and the known configuration of the camera, the picket robot's full pose with respect to the environment can be estimated.

\section{E. Classification}

Slippery terrain is remotely detected by the main robot by using the picket robot as a remote probe to explore the environment. The main robot collects 6 DoF information about the motion of the picket robot, as it tracks its progress across the test surface. At the same time, the picket robot collects proprioceptive data about itself as it maneuveres across the terrain. The dynamics of the VelociRoACH are comprised of repeatable periodic oscillations [14]. We predict that the locomotion dynamics of the picket robot (VelociRoACH) are perturbed by slippery terrain, allowing the dynamic signature of the picket robot to classify low friction regions.

A set of features which describe the locomotion dynamics of the picket robot is therefore needed. To allow for the highest possible rate of terrain classification, these features should be fast to compute and should require the minimum possible sampling period. We chose the features to be the second, third and fourth statistical moments (variance, skew, kurtosis, respectively) of a subset taken from available data. The $k$-th statistical moment, $\mu_{k}^{x}$, of a $n$ length time-series of observations of $x$ is given by

$$
\mu_{k}^{x}=\frac{1}{n} \sum_{i=1}^{n} x_{i}^{k} .
$$

The fourth moment of the observed pitch angle $\theta$ for example, would be denoted $\mu_{4}^{\theta}$.

Features calculated in this fashion have been recently used [10] to successfully classify (94\% accuracy) diverse terrain (tile, carpet, gravel), and were found to be more descriptive than FFT-based [29] features. Observations of the 6 DoF state of the picket robot are used to calculate the features. The main robot uses camera measurements $(x, y, z$

\footnotetext{
${ }^{6}$ Logitech HD Pro Webcam C920
} 


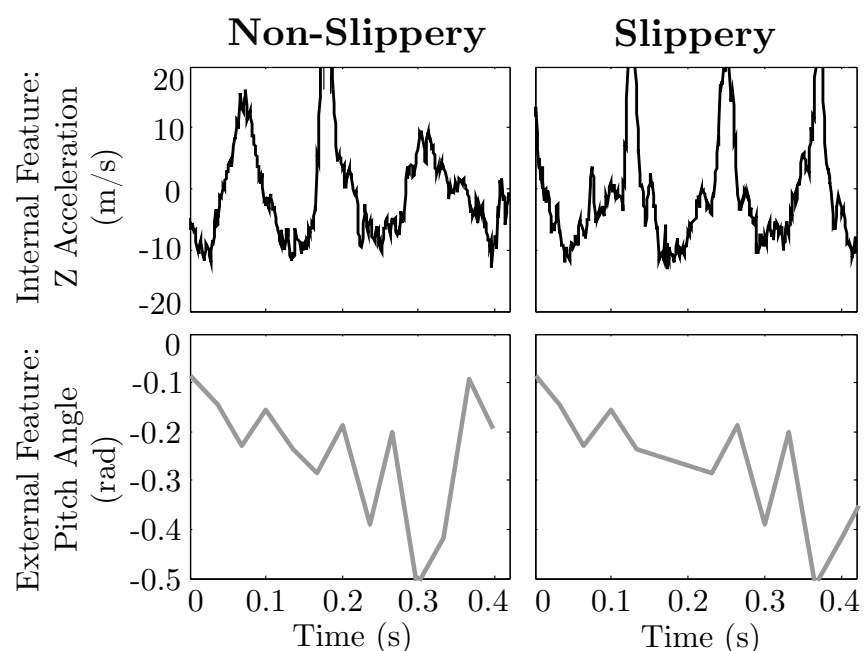

Fig. 4: Time trajectories of internal and external data, for slippery and non-slippery terrain. The internal data is sampled at $1000 \mathrm{~Hz}$, external camera measurements are taken at $30 \mathrm{~Hz}$.

position, $\psi, \theta, \phi$ Euler angles), whereas the picket robot uses measurements from its 6-axis $\operatorname{IMU}(\ddot{x}, \ddot{y}, \ddot{z}$ accelerations, $\dot{\omega}_{1}, \dot{\omega}_{2}, \dot{\omega}_{3}$ rotation rates). An example of the data from which these features are calculated from is shown in Fig. 4.

Support Vector Machines (SVMs) are used to identify slippery terrain using the tabulated features. We use the MATLAB implementation of LIBSVM [6] to do the classification, withholding $25 \%$ of the available data to test the accuracy of the classifier.

Three different SVMs are built to test how well slippery terrain could be classified using different approaches. The first is the "Internal Classifier", which uses 18 proprioceptive features collected from the picket robot. The second is the "External Classifier", which only uses the 18 features collected from the main robot's camera. The third is the "Joint Classifier" which uses the collaborative set of all of the above features. All features are normalized before being used in a soft-margin SVM. The performance of these classifiers is given in Section III-B.

\section{RESULTS}

\section{A. Localization}

Precise localization between the main and the picket robot is required to meaningfully map out the slippery and non-slippery regions of the terrain. Presented first are the results for an isolated visual tracking experiment, which are followed by the localization results for the combined estimation of both robots.

Fig. 5 shows the results for the estimated pose of the picket robot relative to the main robot. To isolate the tracking procedure, we kept the main robot (and hence the camera) stationary to get a fixed transformation between the global and the main robot's coordinate system. In this experiment, the picket robot runs along the main robot's $\tilde{x}$-axis from the lower to the upper camera image border. The estimated position through the visual tracker is satisfactorily accurate when compared to the ground truth data. The root mean
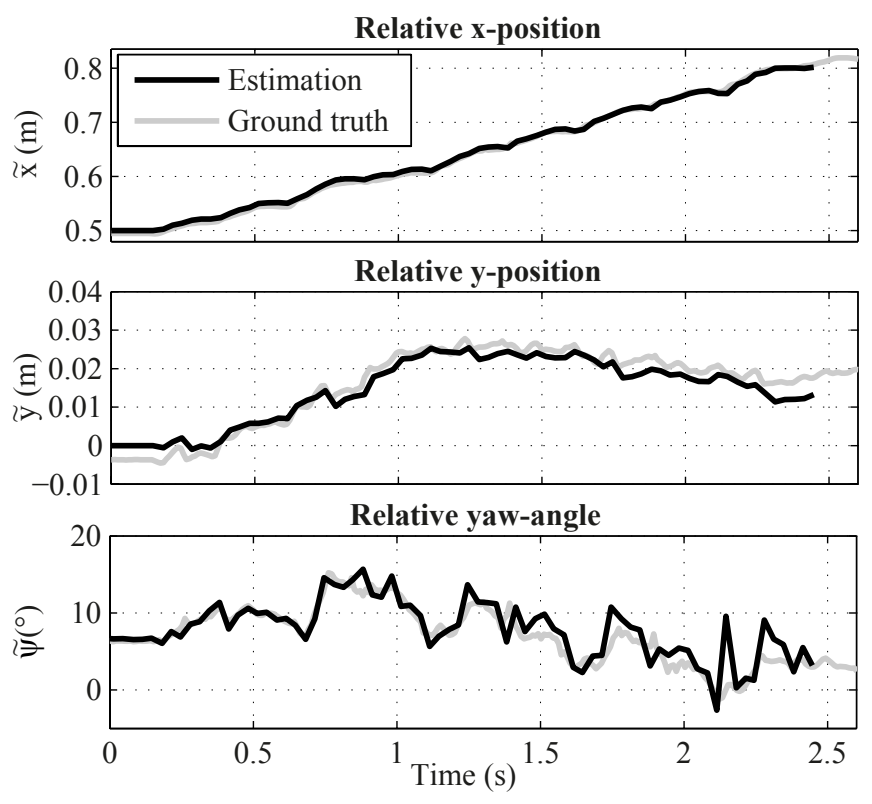

Fig. 5: Evaluation data for the visual tracking performance. For this experiment, the main robot/camera was kept stationary and the picket robot ran along the local $\tilde{x}$-axis (relative position) from the lower to the upper image border.

squared error (RMSE) is $5 \mathrm{~mm}$ for the relative position and $2^{\circ}$ for the relative yaw angle.

Results for the combined localization are shown in Fig. 6. The picket robot is controlled to run in front of the main robot at a constant distance. The main robot localizes itself with the on-board state estimation. The position of the picket robot with respect to the environment is estimated through the transformation chain of the main robot's pose estimation and the visual tracking of the marker. The position error for the main robot is $6.7 \mathrm{~cm}$ after a travel distance of $2 \mathrm{~m}$ (in $18 \mathrm{~s}$ ). The position error for the picket robot for the same experiment is $10.9 \mathrm{~cm}$, which is caused by the cumulative error from on-board state estimation and visual tracking. ${ }^{7}$ Clearly, with our estimation setup, the global positioning error increases over travelled distance. However, our approach does not rely on an absolute global position, but rather on a localized estimate of relative position around the main robot and picket robot. This information is sufficient for the main robot to plan a path avoiding slippery patches on the terrain. In this respect, the presented precision of our method is regarded as sufficient.

\section{B. Classification}

The minimum necessary sampling period (for a given accuracy threshold) is one of the major figures of merit for the application of this classifier. It limits how quickly the teams of robots can traverse terrain while mapping friction properties, and also limits the minimum detectable size of a patch of slippery terrain. Fig. 7 shows the accuracy of the three classifiers as a function of sampling period. The features for the internal classifier are calculated using data

\footnotetext{
${ }^{7}$ The cumulative error is highly sensitive to the error in yaw rotation of the main robot, which is $1^{\circ}$ after the entire travel distance in this experiment.
} 


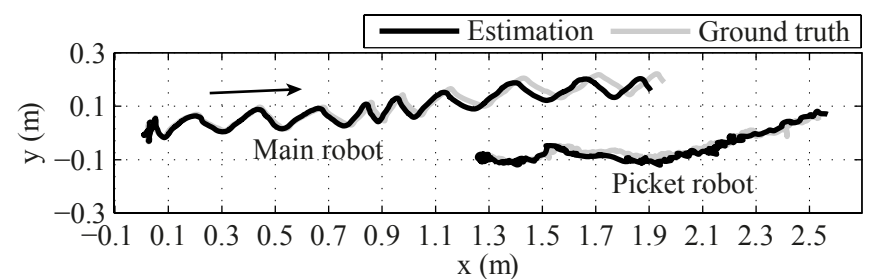

Fig. 6: The main robot walks for $2 \mathrm{~m}$ while the picket robot is controlled to move in front of it at a constant distance. After the entire travel distance of $2 \mathrm{~m}$ the position error is $6.7 \mathrm{~cm}$ for the main robot and $10.9 \mathrm{~cm}$ for the picket robot.

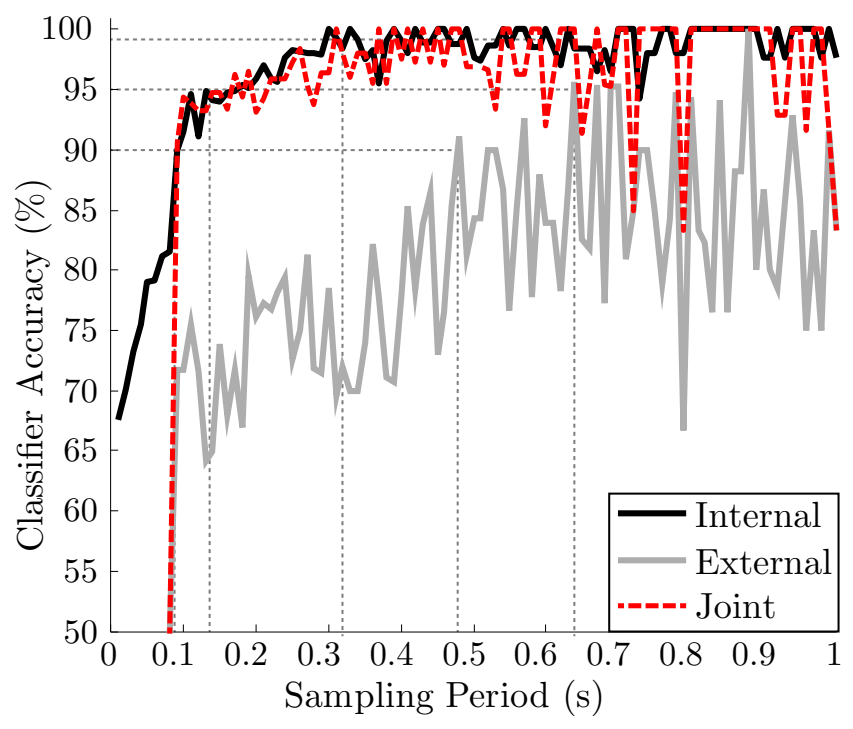

Fig. 7: Accuracies of the classifiers, as a function of sampling period.

collected at more than 30 times the sampling rate of the external measurements. We therefore expect the internal classifier to achieve higher accuracy at a lower period than the external classifier. This result is shown in Fig. 7.

In order to develop classifiers which could be run faster, we used Principal Component Analysis (PCA) on the feature data to identify a small set of highly descriptive features. Previous work in terrain classification has used this approach to the reduce the dimensionality of the feature space [12]. Table I gives the accuracy of the three classifiers when they are restricted a small subset of the most descriptive features. For each classifier in Table I, we give data on classifiers of rank 1 through 4 . The accuracy and dimension of the test set are given in the third column, and the last column gives the features for each classifier in order of expected importance.

As shown in Table I, the accuracy of the classifiers increases as more features are used to detect the slipperiness of the terrain. It should be noted that these classifiers were trained from the same dataset. This means that the External Classifier, which requires twice the sampling period of the Joint and Internal Classifiers, is only able to train and test on a dataset of approximately half the size. The Joint Classifier has the best performance when using fewer features, and is the only one to achieve an accuracy of over $90 \%$ when four or less features are used. The rank-4 Joint Classifier uses features from both the internal and external sets, which
TABLE I: REDUCED RANK APPROXIMATION

\begin{tabular}{cccl}
\hline $\begin{array}{c}\text { Classifier } \\
\text { (Sampling Window) }\end{array}$ & Rank & Accuracy & Features (F) \\
\hline & 1 & $58.0 \%(76 / 131)$ & $\mu_{2}^{\omega_{2}}$ \\
Internal & 2 & $72.5 \%(95 / 131)$ & $\mu_{2}^{\ddot{y}} \mu_{2}^{\omega_{1}}$ \\
$(0.31 \mathrm{~s})$ & 3 & $73.3 \%(96 / 131)$ & $\mu_{2}^{\ddot{y}} \mu_{2}^{\omega_{1}} \mu_{2}^{\ddot{z}}$ \\
& 4 & $81.7 \%(107 / 131)$ & $\mu_{2}^{\omega_{3}} \mu_{2}^{\ddot{z}} \mu_{4}^{\omega_{3}} \mu_{2}^{\ddot{y}}$ \\
\hline & 1 & $67.9 \%(53 / 78)$ & $\mu_{2}^{y}$ \\
& 2 & $64.1 \%(50 / 78)$ & $\mu_{2}^{y} \mu_{4}^{x}$ \\
External & 3 & $83.3 \%(65 / 78)$ & $\mu_{2}^{\theta} \mu_{2}^{y} \mu_{4}^{x}$ \\
$(0.60 \mathrm{~s})$ & 4 & $75.6 \%(60 / 78)$ & $\mu_{2}^{\theta} \mu_{4}^{x} \mu_{2}^{x} \mu_{2}^{y}$ \\
& 1 & $81.1 \%(109 / 134)$ & $\mu_{2}^{\omega_{2}}$ \\
& 2 & $81.1 \%(109 / 134)$ & $\mu_{2}^{\ddot{z}} \mu_{3}^{\ddot{z}}$ \\
& 3 & $84.0 \%(113 / 134)$ & $\mu_{2}^{\theta} \mu_{2}^{\ddot{z}} \mu_{3}^{\ddot{z}}$ \\
Joint & 4 & $92.9 \%(125 / 134)$ & $\mu_{2}^{\theta} \mu_{2}^{\ddot{z}} \mu_{3}^{\ddot{z}} \mu_{2}^{\omega_{1}}$ \\
\hline
\end{tabular}

allowes it to better classify slippery terrain.

Several features are repeatedly chosen most effective for slippery terrain detection. The variance of the $y$ acceleration and position $\left(\mu_{2}^{\ddot{y}}, \mu_{2}^{y}\right)$ is much greater for running the VelociRoACH on the low friction surface, which allows for more lateral motion than other terrains. The variance of the pitch rate and angle $\left(\mu_{2}^{\omega_{2}}, \mu_{2}^{\theta}\right)$ is significantly less for the low friction case. The robot tends to stub its front legs when it is being driven with aperiodic differential steering, the low friction terrain reduces these impacts and thereby reduces the pitch disturbances they cause.

\section{CONCLUSION}

This work developed a framework for remote terrain detection and demonstrated its feasibility with a proof of concept experiment. This proposed framework has four main pieces, a main robot (1) which is assisted by one or more picket robots (2). The main robot has a method to localize itself and the picket robots (3), and the picket robots have a method to classify terrain (4). To demonstrate the concept we used the legged quadruped, StarlETH, as the main robot, and VelociRoACH, as the picket robot.

We demonstrated that a legged odometer based on onboard state estimation is sufficiently accurate to localize the StarlETH in a relevant portion of the global frame near a patch of slippery terrain. The StarlETH is able to locate the VelociRoACH using visual tracking, and give it position feedback to remotely guide it to specific portions of the terrain for classification. This type of joint perception is advantageous because the picket robot does not have the same capability for internal state estimation or remote sensing as the main robot.

For the fourth part of the framework, we tested three different types of terrain classifier, all of which could achieve an accuracy of over $90 \%$ when identifying slippage of the picket robot. Instead of traditional slippage detection methods, we implemented a SVM based terrain classifier, which can be readily extended to identify other types of hazardous terrain. The External Classifier, which used only features tabulated from camera tracking data of the picket 
robot, can achieve an accuracy of $94 \%$ with a sampling period of 0.60 seconds. The Internal Classifier, which only uses features tabulated from the internal IMU of the picket robot, achieves an accuracy of $98 \%$ with just a 0.31 second sampling window. Using the full feature space, the Joint Classifier has a similar performance to the internal classifier. However, the joint classifier distinguished itself as most effective for a light-weight classifier. Using PCA, we chose a subset of features which are expected to be most effective at separating the data. Of all of these low-rank classifiers, only the rank-4 joint classifier was able to achieve an accuracy of over $90 \%$ by using features from both the internal and external sets.

\section{A. Future Work}

This proof of concept work used one picket robot driven directly in front of one main robot. When the team uses this running configuration, it is possible that hazardous terrain which threatens the main robot would not be detected. This problem could be solved using area coverage [7] wherein a robot or team of robots completely canvas an area of interest. Complete coverage would be necessary if the main robot was a traditional wheeled robot, but legged robots such as StarlETH have the ability to discretize terrain into distinct footholds. This reduces the problem of area coverage to one of probabilistic coverage as has been used for robotic demining [1]. Only the terrain properties of the future planned footholds need to be checked, greatly reducing the time the picket robots need to spend mapping the terrain. Future work will explore path planning algorithms and picket robot formations which will more effectively detect hazardous terrain.

\section{ACKNOWLEDGMENTS}

Thanks to the members of the Biomimetic Millisystems Lab for their helpful comments and discussions. Thanks also to the Legged Robotics Team at the Autonomous Systems Lab for their help and support with the experiments.

\section{REFERENCES}

[1] E. U. Acar, H. Choset, Y. Zhang, and M. Schervish, "Path Planning for Robotic Demining: Robust Sensor-Based Coverage of Unstructured Environments and Probabilistic Methods," The International Journal of Robotics Research, vol. 22, no. 7-8, pp. 441-466, Jul. 2003.

[2] S. S. Baek, F. L. Garcia Bermudez, and R. S. Fearing, "Flight control for target seeking by 13 gram ornithopter," 2011 IEEE/RSJ Int. Conf. on Intelligent Robots and Systems, pp. 2674-2681, Sep. 2011.

[3] M. Bloesch, M. Hutter, M. A. Hoepflinger, S. Leutenegger, C. Gehring, C. D. Remy, and R. Siegwart, "State Estimation for Legged Robots Consistent Fusion of Leg Kinematics and IMU," in Robotics: Science and Systems Conference (RSS), 2012.

[4] A. D. Buchan, D. W. Haldane, and R. S. Fearing, "Automatic identification of dynamic piecewise affine models for a running robot," 2013 IEEE/RSJ International Conference on Intelligent Robots and Systems, pp. 5600-5607, Nov. 2013.

[5] R. Castano, R. Manduchi, and J. Fox, "Classification experiments on real-world texture," Third Workshop on Empirical ..., 2001.

[6] C.-J. Chang, Chih-Chung and Lin, "LIBSVM: A library for support vector machines," ACM Transactions on Intelligent Systems and Technology, vol. 2, no. 3, pp. 1--27, 2011.

[7] H. Choset, "Coverage for roboticsA survey of recent results," Annals of mathematics and artificial intelligence, pp. 113-126, 2001.
[8] F. Dellaert, T. Balch, M. Kaess, R. Ravichandran, F. Alegre, M. Berhault, R. McGuire, E. Merrill, L. Moshkina, and D. Walker, "The Georgia Tech Yellow Jackets: A Marsupial Team for Urban Search and Rescue." in AAAI Mobile Robot Competition, 2002.

[9] M. Fiala, "ARTag, a fiducial marker system using digital techniques," in Conference on Computer Vision and Pattern Recognition (CVPR), vol. 2. IEEE, 2005, pp. 590-596.

[10] F. L. Garcia Bermudez, R. C. Julian, D. W. Haldane, P. Abbeel, and R. S. Fearing, "Performance analysis and terrain classification for a legged robot over rough terrain," 2012 IEEE/RSJ International Conference on Intelligent Robots and Systems, pp. 513-519, Oct. 2012.

[11] C. Gehring, S. Coros, M. Hutter, M. Bloesch, M. A. Hoepflinger, and R. Siegwart, "Control of Dynamic Gaits for a Quadrupedal Robot," IEEE International Conference on Robotics and Automation (ICRA), 2013.

[12] P. Giguere and G. Dudek, "Clustering sensor data for autonomous terrain identification using time-dependency," Autonomous Robots, vol. 26, no. 2-3, pp. 171-186, Mar. 2009.

[13] _ _ "A Simple Tactile Probe for Surface Identification by Mobile Robots," IEEE Transactions on Robotics, vol. 27, no. 3, pp. 534-544, Jun. 2011.

[14] D. W. Haldane, K. C. Peterson, F. L. Garcia Bermudez, and R. S. Fearing, "Animal-inspired Design and Aerodynamic Stabilization of a Hexapedal Millirobot," IEEE Int. Conf. on Robotics and Automation, 2013.

[15] M. a. Hoepflinger, C. D. Remy, M. Hutter, L. Spinello, and R. Siegwart, "Haptic terrain classification for legged robots," 2010 IEEE International Conference on Robotics and Automation, pp. 2828-2833, May 2010.

[16] A. M. Hoover and R. S. Fearing, "Fast scale prototyping for folded millirobots," IEEE Int. Conf. on Robotics and Automation, pp. 886892, 2008.

[17] M. Hutter, C. Gehring, M. Bloesch, M. A. Hoepflinger, C. D. Remy, and R. Siegwart, "StarlETH: A compliant quadrupedal robot for fast, efficient, and versatile locomotion," in Proceedings of the International Conference on Climbing and Walking Robots (CLAWAR), 2012.

[18] K. Jayaram, J. M. Mongeau, B. McRae, and R. J. Full, "High-speed horizontal to vertical transitions in running cockroaches reveals a principle of robustness," in Society for Integrative and Comparative Biology, 2010.

[19] A. Milella, F. Pont, and R. Siegwart, "Model-based relative localization for cooperative robots using stereo vision," in Conference on Mechatronics and Machine Vision. IEEE, 2005.

[20] R. R. Murphy, "Marsupial and shape-shifting robots for urban search and rescue," IEEE Intelligent Systems and their Applications, vol. 15, no. 2, pp. 14-19, 2000.

[21] L. Parker and B. Kannan, "Tightly-coupled navigation assistance in heterogeneous multi-robot teams," in International Conference on Intelligent Robots and Systems (IROS). IEEE, 2004.

[22] L. E. Parker, "Adaptive heterogeneous multi-robot teams," Neurocomputing, vol. 28, no. 1-3, pp. 75-92, Oct. 1999.

[23] _ - "Multiple Mobile Robot Systems," in Springer Handbook of Robotics, B. Siciliano and O. Khatib, Eds. Berlin, Heidelberg: Springer Berlin Heidelberg, 2008, pp. 921-941.

[24] K. Rainio, "ALVAR A Library for Virtual and Augmented Reality Users Manual (v.2.0)," VTT Technical Research Centre of Finland, Tech. Rep., 2012.

[25] P. R. Sinhat and R. K. Bajcsy, "Robotic Exploration of Surfaces and its Application to Legged Locomotion," IEEE Int. Conf. on Robotics and Automation, pp. 221--226, 1992.

[26] D. Vail and M. Veloso, "Learning from accelerometer data on a legged robot," Proceedings of the 5th IFACEURON Symposium on Intelligent Autonomous Vehicles, 2004.

[27] N. Vandapel and D. Huber, "Natural terrain classification using 3-d ladar data," ... and Automation, 2004. ..., no. April, 2004.

[28] C. Weiss, H. Tamimi, and a. Zell, "A combination of vision- and vibration-based terrain classification," 2008 IEEE/RSJ International Conference on Intelligent Robots and Systems, pp. 2204-2209, Sep. 2008.

[29] C. Weiss, H. Fr, and A. Zell, "Vibration-based Terrain Classification Using Support Vector Machines,” pp. 4429-4434, 2006. 